\title{
The effect of antenatal care follow-up on neonatal health outcomes: a systematic review and meta-analysis
}

\author{
Amsalu Taye Wondemagegn ${ }^{1 *}$, Animut Alebel ${ }^{2}$, Cheru Tesema ${ }^{3}$ and Worku Abie ${ }^{1}$
}

\author{
* Correspondence: \\ 50amsalu@gmail.com \\ ${ }^{1}$ Department of Biomedical \\ Sciences, School of Medicine, Debre \\ Markos University, Debre Markos, \\ Ethiopia \\ Full list of author information is \\ available at the end of the article
}

\begin{abstract}
Background: Neonatal mortality is one of the major public health problems throughout the world and most notably in developing countries. There exist inconclusive findings on the effect of antenatal care visits on neonatal death worldwide. Thus, the aim of this systematic review and meta-analysis was to reveal the pooled effect of antenatal care visits on neonatal death.

Methods: The present systematic review and meta-analysis was performed using published literature, which was accessed from national and international databases such as, Medline/PubMed, EMBASE, CINAHL, Cochrane Central library, Google Scholar, and HINARI. STATA/SE for windows version 13 software was used to calculate the pooled effect size with 95\% confidence intervals $(95 \% \mathrm{Cl}$ ) of maternal antenatal care visits on neonatal death using the DerSimonian and Laird random effects meta-analysis (random effects model), and results were displayed using forest plot. Statistical heterogeneity was checked using the Cochran $Q$ test (chi-squared statistic) and $P^{2}$ test statistic and by visual examination of the forest plot.
\end{abstract}

Results: A total of 18 studies, which fulfilled the inclusion criteria, were included in the present systematic review and meta-analysis. The finding of the present systematic review and meta-analysis revealed that antenatal care visits decrease the risk of neonatal mortality [pooled effect size $0.66(95 \% \mathrm{Cl}, 0.54,0.80)$ ]. Cochrane $Q$ test $(P<0.001)$ revealed no significant heterogeneity among included studies, but $P^{2}$ statistic revealed sizeable heterogeneity up to $80.5 \%\left(P^{2}=80.5 \%\right)$. In the present metaanalysis traditional funnel plot, Egger's weighted regression $(P=0.48)$ as well as Begg's rank correlation statistic $(P=0.47)$ revealed no evidence of publication bias.

Conclusions: The present systematic review and meta-analysis revealed that antenatal care visits were significantly associated with lower rates of neonatal death. The risk of neonatal death was significantly reduced by $34 \%$ among newborns delivered from mothers who had antenatal care visits. Thus, visiting antenatal care clinics during pregnancy is strongly recommended especially in resource-limited settings like countries of sub-Saharan Africa.

Keywords: Antenatal care, Maternal health service, Follow up, Visits, Utilizations, Neonatal mortality/death, Meta-analysis 


\section{Background}

Neonatal mortality is defined as the death of a newborn in the first 4 weeks of life (neonatal period), and it is expressed in terms of rate of neonatal deaths per 1000 live births [1]. Neonatal mortality is one of the major public health problems throughout the world, most notably in developing countries. Globally, an estimated number of 2.6 million neonatal deaths occurred in 2016, accounting for $46 \%$ of deaths among under-five children [2, 3]. Almost all (99\%) newborn deaths occur in low- and middle-income countries. Moreover, Africa and South Asia have made the least progress in reducing neonatal deaths [1]. Sub-Saharan Africa (SSA) carries the highest neonatal mortality in the world and achieved the lowest progress in the reduction of neonatal mortality [4].

One of the targets of the Sustainable Development Goals (SDGs) fixed by the United Nations in 2015 is to end preventable deaths of newborns, with all countries aiming to reduce neonatal mortality to as low as 12 deaths per 1000 live births by the year 2030 [2]. Globally, neonatal mortality declined from 5.1 million in 1990 to 2.6 million in 2016, but this decline in neonatal mortality over 1990-2016 has been slower than that of post-neonatal under-five mortality (1-59 months): 47\%, compared with $58 \%$ globally. This pattern applies to most low- and middle-income countries [2]. Evidence suggests that about $75 \%$ of neonatal deaths in developing countries could be prevented with simple and low-cost tools that already exist, such as antibiotics for pneumonia and sepsis, sterile blades to cut umbilical cords, and using knit caps and kangaroo care to keep babies warm [5,6]. These modifiable risk factors could be avoided through implementation of preventive measures like antenatal care (ANC) services [7].

ANC is one of the fundamental strategies recommended to reduce the risk of neonatal mortality in any community, despite socio-demographic background [8-11]. ANC improves the survival and health of babies directly by reducing stillbirths and neonatal deaths and indirectly by providing an entry point for health contacts with the mother at a key point in the continuum of care [12]. In addition, it will help the health professionals to identify women at increased risk of adverse pregnancy outcomes. The World Health Organization (WHO) recommends at least eight ANC visits to provide effective ANC services, particularly in low-income countries [13].

In different parts of the world, several studies have been conducted to determine the effect of ANC on neonatal mortality. The findings reported from these studies were controversial and inconclusive in nature. In most studies, providing ANC services reduced the risk of neonatal mortality. In others, the ANC visits were not significantly associated with neonatal mortality. For better intervention, current and up-to-date information regarding the effect of ANC on neonatal survival is crucial, especially in low- and middle-income countries where most neonatal deaths occur. However, in recent years, despite small studies, there has been no worldwide study to determine the effect of ANC on neonatal mortality. Therefore, this systematic review and meta-analysis aims to estimate the effect of ANC follow-up on neonatal mortality using available studies. The findings from this systematic review will highlight the effect of ANC interventions, with implications to improve health workers' operations and cost-effectiveness and to accelerate the reduction of neonatal deaths. 


\section{Methods}

\section{Searching strategies}

The present systematic review and meta-analysis was conducted to quantify the pooled effect of ANC on neonatal mortality. Literature was reviewed from national and international databases. The following databases were systematically searched: Medline/ PubMed, EMBASE, CINAHL, Cochrane Central library, Google Scholar, and HINARI (Health Inter Network Access to Research Initiative) from August 29 to October 30, 2017. The reports were accessed using the following key terms/like Mesh terms, Emtree/: "antenatal care/ANC," "maternal health service," "follow up," "visits," "utilizations," "neonatal death," and "newborn care." The key terms were used individually and in combination through "AND" and "OR." In addition, after identification of studies and review articles, their lists of reference were searched to identify more eligible studies. The above database search strategy and terms are presented in (Additional file 1: Table S1). This systematic review and meta-analysis used the PICO (Population, Intervention, Comparison and Outcomes) framework to determine the eligibility of the articles included. The study Population (P) were neonates (age $<28$ days), the Intervention (I) was focused ANC follow-up, the Comparison (C) group were neonates born from mothers who did not have ANC follow-up, and the Outcomes $(\mathrm{O})$ of this study were the occurrence of death within 28 days after delivery.

\section{Study selection}

Potentially eligible studies for this systematic review and meta-analysis were selected in three stages: titles alone, abstracts, and then full-text articles, based on inclusion criteria: All quantitative studies reported in English language, published in peer-reviewed journals, and revealed the association between antenatal care visits and neonatal deaths were included.

However, studies which did not report the maternal ANC visit status as well as outcomes of their newborns were excluded. In addition, articles, which were not fully accessible, after at least two-email contact with the primary authors, were excluded. Exclusion of these articles is because of the inability to assess the quality of articles in the absence of full text.

Moreover, review articles, commentary, and editorials were excluded from analysis, but read to identify eligible articles for the current review. Potentially eligible articles were identified by two reviewers (ATW and AA), through independent reading of the titles and abstracts, which were searched and accessed broadly. The full texts of these articles were accessed, and independent assessment was carried out by two reviewers, ATW and AA, for eligibility based on the predetermined inclusion and exclusion criteria. Discrepancies between the reviewers were resolved through discussion and common consensus of all investigators. Multiple publications of the same study were not identified.

\section{Data abstraction and quality assessment of the studies}

Two reviewers (ATW and AA) independently extracted data, which was then confirmed by the other investigators (CT and WA). The data extraction was performed using the following template: first author, year of publication, study setting, study design, sample size, number of survivors and died neonates among ANC visitor mothers, 
and number of survivors and died neonates among those mothers without ANC visits. Controversies during the data extraction process were resolved through discussion and common understanding among the reviewers. To the researchers' knowledge, there exist no well-defined tools for assessing quality of observational epidemiological studies [14]. However, sample size, sampling method, and response rate were considered to assess the quality of included studies. The studies reported using larger sample size (reported outcomes on at least 50 participants), random sampling, and higher response rate (studies with response rate greater than $80 \%$ ) were considered as high-quality studies.

\section{Statistical analysis}

Data were extracted from each eligible article using a template prepared in Microsoft Excel spreadsheet software and imported into STATA/SE for windows Version 13 software for further analysis. Stata version 13 was used to calculate the pooled effect size with 95\% confidence intervals (95\% CI) of maternal ANC visit status on neonatal death using the DerSimonian and Laird [15] random effects meta-analysis (random effects model).

\section{Assessing statistical inconsistency and publication bias}

Statistical heterogeneity was weighed using the Cochran $Q$ test (chi-squared statistic) and $I^{2}$ test statistic and by visual examination of the forest plot (overlap of confidence intervals). Cochran's $Q$ test was used to test the null hypothesis of no significant heterogeneity across the studies [16]. Cochran's $Q$ is calculated as the weighted sum of squared differences between individual study effects and the pooled effect across studies, with the weights being those used in the pooling method. Cochran's $Q$ statistic follows a chi-squared distribution with $k-1$ degree of freedom where $k$ is the number of studies. Cochran's $Q$ statistical heterogeneity test is considered as statistically significant at $P<0.10$. The $I^{2}$ statistic, the percentage of variation (inconsistency) in the measures of association across studies that is due to heterogeneity rather than chance, [17] was also estimated. The $I^{2}$ statistic is equal to the quantity of Cochran's $Q$ minus its degree of freedom (df) divided by Cochran's $Q$ times $100 \%$, or $I^{2}=100 \% \times(Q-\mathrm{df}) / Q$. The value of $I^{2}$ ranges between 0 and $100 \%$, where $0 \%$ indicates no observed heterogeneity and large values indicate increasing heterogeneity [17]. An $I^{2}$ value of $25 \%, 50 \%$, and $75 \%$ is considered as low, moderate, and high heterogeneity [17]. Each study's risk ratio (RR) with 95\% confidence intervals (CIs) was presented on traditional funnel plots and Begg's funnel plot to reveal publication bias. In addition, Egger's weighted regression and Begg's rank correlation tests were used to check for publication bias $(P<0.05$ is considered statistically significant). Cumulative meta-analysis was also run to assess the effect of each study on the pooled estimate.

\section{Results}

\section{Accessed studies}

The reports of the present meta-analysis was presented based on the Preferred Reporting Items for Systematic Reviews and Meta-Analyses (PRISMA) guideline [18] (Additional file 2: Table S2). A total of 594 articles related to the review titles were accessed in our initial literature search. We removed duplicate retrievals, and 494 articles remained. Upon initial screening, 444 articles were excluded by their titles which 
were found to be non-pertinent because of one of the following reasons: the titles of most of the papers were not directly related to the present topic, the titles of some of the papers consider an individual predictor other than ANC follow-up as exposure variable for neonatal health, and the titles of the remaining paper were for reviews in other topic areas. Of the remaining 50 articles, abstracts were screened and 20 articles were excluded because maternal health service utilization status was not considered as one of the exposure variables (predictor) on child health. For the remaining 30 papers, full-text articles were accessed and evaluated for eligibility based on predetermined inclusion and exclusion criteria and 12 studies were excluded. This was due to some of the articles only reporting the effect of delivery status and postnatal care on neonatal health status and other reports using infant and perinatal mortality as an outcome variable. Finally, 18 studies that fulfilled the inclusion criteria were included in the present systematic review and meta-analysis (Fig. 1).

\section{Description of background characteristics and outcome variables of included studies}

Regarding study design, half (9) of the studies included in this systematic review and meta-analysis were cross-sectional studies [19-27], four were case-control studies [28-31], four cohort studies [32-35], and one community trial study [36]. The publication year of the included studies ranged between 2009 and 2017. The sample size of individual included studies ranged from 198 in a case-control study in Nepal to 56,307 in a cross-sectional study in Nigeria. Among the 18 included studies, almost all (94\%) were from African and Asian low- and middle-income countries. Twelve studies were from

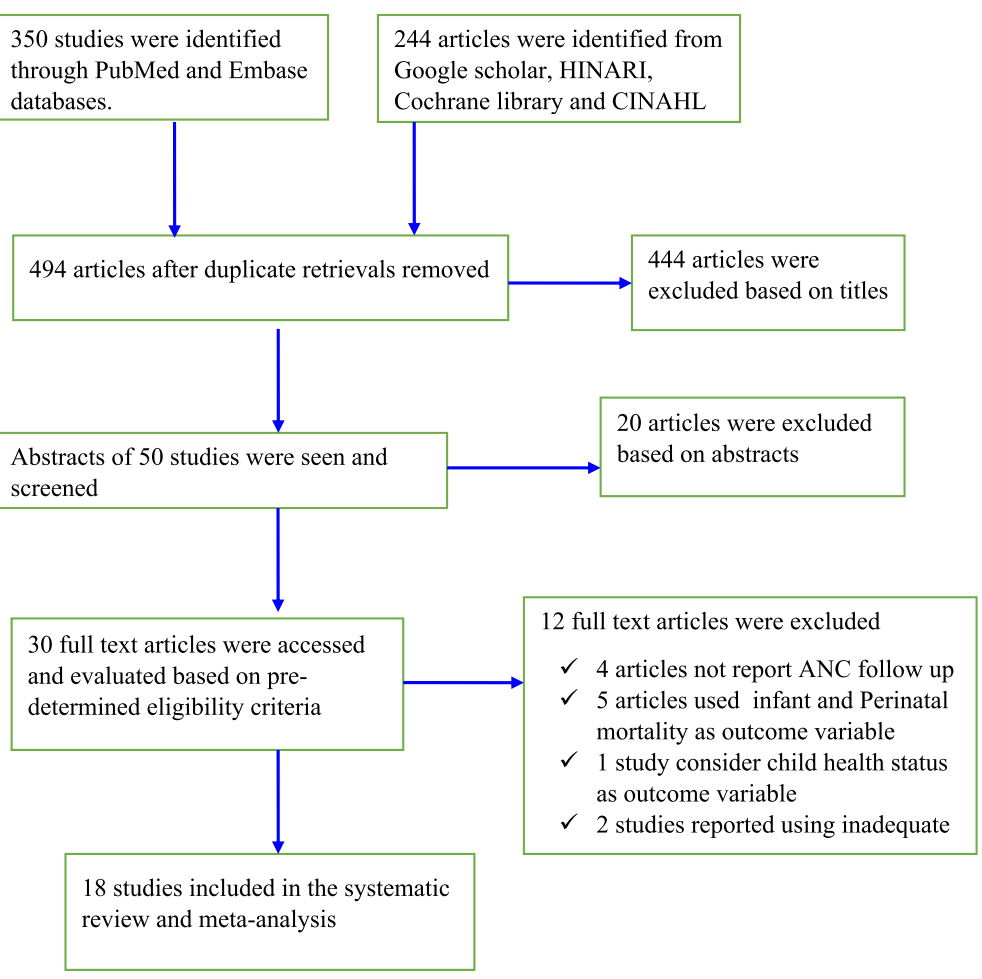

Fig. 1 Flow chart, which reveals the procedures of study selection for the current systematic review and meta-analysis 
SSA: Ethiopia [19, 31-33, 35], Kenya [26, 28], Malawi [22], Burkina Faso [36], Cameroon [34], Uganda [23], and Nigeria [24]. Five studies were from Asia: India [21], Iraq [27], Nepal [20, 29], and Pakistan [25]. Only one study was obtained from South America, Brazil [30]. In the current meta-analysis, a total of 94,118 live births were involved. From all live births, 3097 died within 28 days of birth, with a neonatal mortality rate of 32.91 per 1000 live births, and the remaining 91,021 survived beyond the first 28 days after birth. The total number of live births born from mothers who did not attend ANC visits was 49,706, and the total number of live births born to mothers who attended ANC follow-up was 44,412. Further descriptions of the characteristics and outcomes of this systematic review and meta-analysis are presented in Table 1 .

\section{Findings of heterogeneity and publication bias of included studies}

Analysis of included studies revealed heterogeneity using the Cochrane $Q$ test statistic $\left[X^{2}=87.2(\mathrm{df}=17) P<0.001\right]$. In addition, sizeable heterogeneity was found to be up to $80.5 \%$ using the $I^{2}$ test statistic $\left(I^{2}=80.5 \%\right)$. Because of this, a random effects meta-analysis model was used to quantify the effect of ANC follow-up on neonatal mortality. The effect estimates were distributed symmetrically on a traditional funnel plot (Fig. 2), indicating that there was no evidence of publication bias. Moreover, to ascertain this Begg's funnel plot (Fig. 3), Begg's rank correlation test was conducted, and the result of the test statistics revealed that there was no statistically significant bias with Kendall's score of -19 and $P=0.47$. More importantly, Egger's weighted

Table 1 Summary of 18 studies included in the meta-analysis to determine the effect of ANC visits on neonatal mortality

\begin{tabular}{|c|c|c|c|c|c|c|c|}
\hline \multirow{2}{*}{$\begin{array}{l}\text { Primary author } \\
\text { (publication year) }\end{array}$} & \multirow{2}{*}{$\begin{array}{l}\text { Study } \\
\text { setting/ } \\
\text { country }\end{array}$} & \multirow[t]{2}{*}{ Study design } & \multirow{2}{*}{$\begin{array}{l}\text { Sample } \\
\text { size }\end{array}$} & \multicolumn{2}{|c|}{ No ANC visits } & \multicolumn{2}{|l|}{ ANC visits } \\
\hline & & & & Survivors & $\begin{array}{l}\text { Neonatal } \\
\text { death }\end{array}$ & Survivors & $\begin{array}{l}\text { Neonatal } \\
\text { death }\end{array}$ \\
\hline Kumar et al. (2014) [21] & India & Cross sectional & 14,293 & 10,978 & 224 & 1824 & 37 \\
\hline Al-Ani et al. (2009) [27] & Iraq & Cross sectional & 3249 & 1925 & 57 & 1242 & 25 \\
\hline Shah et al. (2015) [29] & Nepal & Case-control & 198 & 10 & 17 & 69 & 57 \\
\hline Koffi et al. (2015) [22] & Malawi & Cross sectional & 24,000 & 13,971 & 189 & 9709 & 131 \\
\hline Dahiru T (2015) [24] & Nigeria & Cross sectional & 56,307 & 9196 & 222 & 10,577 & 215 \\
\hline Worku et al. (2014) [33] & Ethiopia & Cohort & 763 & 452 & 10 & 255 & 10 \\
\hline Arunda et al. (2017) [26] & Kenya & Cross sectional & 14,190 & 539 & 24 & 8074 & 87 \\
\hline Nascimento et al. (2012) [30] & Brazil & Case-control & 396 & 70 & 38 & 194 & 94 \\
\hline Paudel et al. (2013) [20] & Nepal & Cross sectional & 12,674 & 1988 & 49 & 2036 & 27 \\
\hline Ayaz et al. (2010) [25] & Pakistan & Cross sectional & 565 & 253 & 4 & 297 & 11 \\
\hline Diallo et al. (2011) [36] & Burkina Faso & Community trial & 1162 & 226 & 12 & 598 & 28 \\
\hline Ndombo et al. (2017) [34] & Cameroon & Cohort & 332 & 86 & 30 & 194 & 22 \\
\hline Yego et al. (2017) [28] & Kenya & Case-control & 600 & 227 & 105 & 133 & 13 \\
\hline Kananura et al. (2016) [23] & Uganda & Cross sectional & 2237 & 1112 & 57 & 866 & 18 \\
\hline Worku et al. (2012) [32] & Ethiopia & Cohort & 3789 & 205 & 106 & 2556 & 732 \\
\hline Wakgari et al. (2013) [19] & Ethiopia & Cross sectional & 17,817 & 5506.4 & 246.9 & 2846.07 & 48.93 \\
\hline Kolola et al. (2016) [31] & Ethiopia & Case-control & 336 & 119 & 46 & 122 & 26 \\
\hline Debelew et al. (2014) [35] & Ethiopia & Cohort & 3604 & 1362 & 44 & 1204 & 34 \\
\hline
\end{tabular}




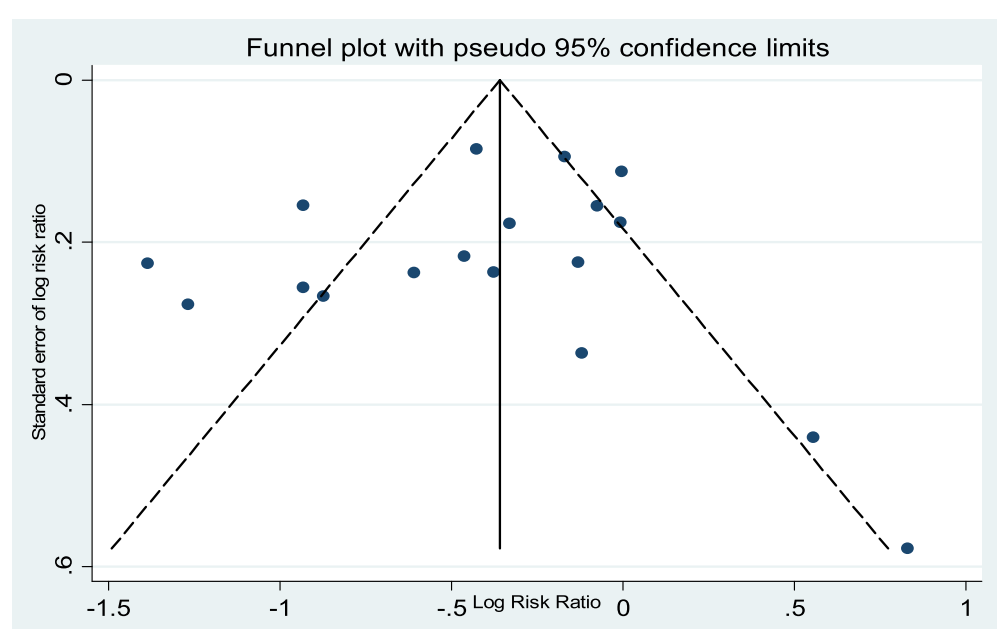

Fig. 2 Traditional funnel plot of 18 included studies of the effect of ANC on neonatal death; the horizontal line refers the effect estimate, and the vertical line refers the expected $95 \%$ confidence intervals

regression test statistic was considered, revealing that there was no significant evidence of publication bias with $r=-0.88(95 \% \mathrm{CI}=-3.47,1.72)$ and $P=0.48$.

\section{The effect of ANC follow-up on neonatal mortality}

The pooled effect size of neonatal death among those live births born to mothers who had ANC visits was $0.66(0.54,0.80)$ compared to those born to mothers without having ANC visits in the random effects model. The finding of the present systematic review and meta-analysis revealed that ANC visits decrease the risk of neonatal mortality by $34 \%$ (Fig. 4).

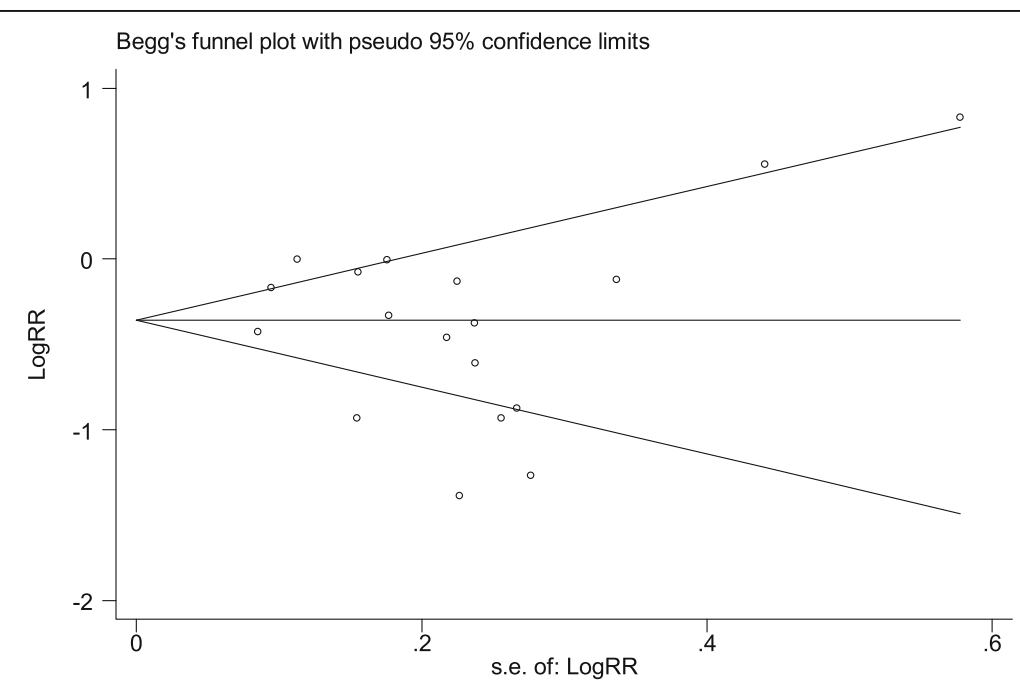

Fig. 3 Begg's funnel plot of 18 included studies of the effect of ANC visits on neonatal death; the horizontal line in the plot refers to the natural logarithm of effect estimate, and the vertical line refers the expected $95 \%$ confidence intervals 
Study

ID .
ES $(95 \% \mathrm{Cl})$

$0.99(0.70,1.40)$

$0.69(0.43,1.09)$

$0.72(0.51,1.02)$

$1.00(0.80,1.24)$

$0.85(0.70,1.02)$

$1.74(0.74,4.13)$

$0.25(0.16,0.39)$

$0.93(0.68,1.26)$

$0.54(0.34,0.87)$

$2.29(0.74,7.12)$

$0.89(0.46,1.72)$

$0.39(0.24,0.65)$

$0.28(0.16,0.48)$

$0.42(0.25,0.70)$

$0.65(0.55,0.77)$

$0.39(0.29,0.53)$

$0.63(0.41,0.97)$

$0.88(0.56,1.36)$

$0.66(0.54,0.80)$

Overall $(\mathrm{I}$-squared $=80.5 \%, p=0.000$ )

NOTE: Weights are from random effects analysis

1

Fig. 4 Forest plot of 18 included studies, which reveal the effect of ANC visits on neonatal death. The size of the square is proportional to the precision of the study-specific effect estimates, and the bars indicate the corresponding $95 \% \mathrm{Cls}$. The diamond is centered on the summary ES of all included studies, and the width indicates the corresponding $95 \% \mathrm{Cl}$

\section{Subgroup analysis}

To decrease sizeable heterogeneity, subgroup analysis was performed based on the study design, sample size, and study settings. Accordingly, in the random effects model, cross-sectional and case-control studies were found to reveal significant effect size and the remaining cohort and community trial studies did not reveal significant effect size. The present meta-analysis also revealed different effect size with different sample size, and the higher the sample size the more precise the effect size. Finally, the setting in which the included studies were conducted was an important variable that contributed to the effect size differences in the random effects model. Studies conducted in SSA revealed statistically significant effect size (Table 2 ).

\section{Discussion}

Globally, neonatal mortality remains a major concern, despite numerous interventions that have been made to improve the survival of newborns in recent years. The current systematic review and meta-analysis is perhaps the first of its kind to be conducted at the global level to examine the effect of ANC on neonatal mortality. The findings of this study will have important implications for maternal and child health programs run by governmental and non-governmental organizations. The findings of this meta-analysis revealed that ANC follow-up has a significant effect on neonatal mortality. In this meta-analysis, as most of the included studies were obtained from low- and middle-income countries, the result could be better applied for these countries. The meta-analysis indicated that neonatal mortality could be reduced by $34 \%$ through the 
Table 2 Subgroup analysis of 18 included studies in this systematic review and meta-analysis considering study design, sample size, and study settings

\begin{tabular}{lll}
\hline Variables used for subgroup analysis & & Random effects RR with $(95 \% \mathrm{Cl})$ \\
\hline Study design & Cross sectional & $0.64(0.46,0.88)$ \\
& Case-control & $0.61(0.40,0.94)$ \\
& Cohort & $0.72(0.48,1.07)$ \\
Community trial & $0.89(0.46,1.72)$ \\
Sample size & $<1000$ & $0.7(0.47,1.05)$ \\
& $1000-10,000$ & $0.67(0.55,0.82)$ \\
Study settings & $>10,000$ & $0.61(0.41,0.9)$ \\
& SSA & $0.59(0.46,0.76)$ \\
& Asia & $0.78(0.58,1.06)$ \\
& South America & $0.93(0.68,1.25)$ \\
\hline
\end{tabular}

implementation of ANC follow-up. This finding is consistent with a Demographic and Health Survey (DHS)-based study conducted in SSA countries to assess antenatal care and newborn survival [37].

It is well-known that ANC visits may help to reinforce maternal education and compliance, and provide an opportunity for screening for warning signs of pregnancy complications and treatment of infections [38]. In addition, ANC provides an important opportunity for health workers to teach mothers how to recognize warning signs of complications during pregnancy, labor, and delivery and encourage them to plan clean and safe deliveries preferably with trained assistance [39, 40]. Furthermore, during ANC follow-up, health care providers can provide information on postpartum care, newborn care, breastfeeding, problem signs, and appropriate action to be taken [41].

In this meta-analysis, to identify the possible sources of heterogeneity, we performed subgroup analysis based on the regions, sample size, and study design. The result of this subgroup analysis noted that the effect of ANC is statistically significant in SSA countries where more than $99 \%$ of worldwide neonatal deaths occurred [1]. The result obtained from this subgroup analysis indicated that $41 \%$ of neonatal mortality in SSA could be prevented through the implementation of ANC. The possible explanation for this variation in the effect of ANC on neonatal mortality might be due to the difference in ANC coverage across the world. In SSA, there is relatively low ANC coverage as compared to other regions of the world [42]. In areas where there is low coverage of ANC, mothers usually did not have access to ANC services. Therefore, pregnant women may not receive adequate health education regarding warning signs of pregnancy complications and often only go to a health facility after encountering difficulties during labor. As a result, infants are more likely to have health problems and die during the neonatal period.

\section{Limitations}

Only English articles or reports were considered to conduct this review. In addition, the nature of the design of the included studies and the adequacy sample size might affect the estimated report. Furthermore, in this meta-analysis, the majority of the included studies were reported from developing countries, especially SSA and Asia. Therefore, the result may only be representative of the above regions. 


\section{Conclusions and recommendations}

The present systematic review and meta-analysis revealed that ANC visits were significantly associated with lower rates of neonatal death. The risk of neonatal death was significantly reduced overall by $34 \%$ among those newborns of mothers who attended ANC visits and more so (41\%) in SSA. Thus, visiting ANC clinics during pregnancy is strongly recommended especially in resource-limited settings such as countries of SSA.

\section{Additional files}

Additional file 1: Table S1. Search strategy for the MEDLINE/PubMed, Embase, Google Scholar and other databases used to access those articles which reveal effect of ANC follow-up on neonatal health outcomes. (DOCX $12 \mathrm{~kb})$

Additional file 2: Table S2. PRISMA checklist. (DOC 65 kb)

Abbreviations

ANC: Antenatal care; Cl: Confidence interval; ES: Effect size; NM: Neonatal mortality; RR: Risk ratio; SSA: Sub-Saharan Africa

Acknowledgements

Not applicable

Funding

No funding was obtained for this study.

Availability of data and materials

Data will be available upon request to the corresponding author.

Authors' contributions

ATW contributed to the conception of research protocol, study design, literature review, data extraction, data analysis, interpretation, and drafting of the manuscript. AAW, CT, and WA contributed to the data analysis, reviewing of the manuscript, data extraction, and quality assessment. All authors have read and approved the manuscript.

Ethics approval and consent to participate

Not applicable

Consent for publication

Not applicable

\section{Competing interests}

The authors declare that they have no competing interests.

\section{Publisher's Note}

Springer Nature remains neutral with regard to jurisdictional claims in published maps and institutional affiliations.

\section{Author details}

${ }^{1}$ Department of Biomedical Sciences, School of Medicine, Debre Markos University, Debre Markos, Ethiopia.

${ }^{2}$ Department of Nursing, College of Health Sciences, Debre Markos University, Debre Markos, Ethiopia. ${ }^{3}$ Department of Public Health, College of Health Sciences, Debre Markos University, Debre Markos, Ethiopia.

Received: 29 December 2017 Accepted: 18 October 2018

Published online: 17 December 2018

\section{References}

1. The partnership for maternal and child health: Newborn death and illness available at http://www.who.int/pmnch/ media/press_materials/fs/fs_newborndealth_illness/en/. 2015.

2. WHO U, United Nations, WORLD BANK GROUP,: Levels \& trends in child mortality available at https://www.unicef.org/ media/files/IGME_Report_Final2.pdf. 2015.

3. WHO: Global Health Observatory (GHO) data available at http://www.who.int/gho/child_health/mortality/en/. 2015.

4. WHO U, United Nations, WORLD BANK GROUP,: Estimates developed by the UN inter-agency Group for Child Mortality Estimation available at https://www.unicef.org/media/files/2013_IGME_child_mortality_Report.pdf. 2013.

5. UNITED NATIONS: The Millennium Development Goals reports available at http://www.un.org/millenniumgoals/2015 MDG_Report/pdf/MDG\%202015\%20rev\%20\%28July\%201\%29.pdf. 2015.

6. Federal Ministry of Health of Ethiopia: Neonatal Intensive Care Unit (NICU) Training Participants' Manual unpublished report 2014.

7. Akinyemi JO, Bamgboye EA, Ayeni O. Trends in neonatal mortality in Nigeria and effects of bio-demographic and maternal characteristics. BMC Pediatr. 2015;15(1):36. 
8. Lambon-Quayefio MP, Owoo NS. Examining the influence of antenatal care visits and skilled delivery on neonatal deaths in Ghana. Appl Health Econ Health Policy. 2014;12(5):511-22.

9. Wehby GL, Murray JC, Castilla EE, Lopez-Camelo JS, Ohsfeldt RL. Prenatal care effectiveness and utilization in Brazil. Health Policy Plan. 2009;24(3):175-88.

10. Singh A, Pallikadavath S, Ram F, Alagarajan M. Do antenatal care interventions improve neonatal survival in India? Health Policy Plan. 2013;29(7):842-8.

11. Gajate Garrido G: The impact of adequate prenatal care in a developing country: testing the WHO recommendations. 2009

12. WHO: Antenatal Care World Health Organization available at http:/www.who.int/pmnch/media/publications/ aonsectionllI_2.pdf.

13. Lawn J, Kerber K: Opportunities for Africa's newborns: practical data policy and programmatic support for newborn care in Africa 2006

14. Sanderson S, Tatt ID, Higgins JP. Tools for assessing quality and susceptibility to bias in observational studies in epidemiology: a systematic review and annotated bibliography. Int J Epidemiol. 2007;36:666-76.

15. DerSimonian RLN. Meta-analysis in clinical trials. Control Clin Trials. 1986;7:177-88.

16. hardy RJTS. Detecting and describing heterogeneity in meta-analysis. Stat Med. 1998;17:841-56

17. Higgins JP, Thompson SG, Deeks JJ, Altman DG. Measuring inconsistency in meta-analyses. BMJ. 2003;327:557-60.

18. Moher D LA, Tetzlaff J, Altman DG Preferred reporting items for systematic reviews and meta-analyses: the PRISMA statement. PLoS Med 2009, 6:e1000097.

19. Wakgari N, Wencheko E. Risk factors of neonatal mortality in Ethiopia. Ethiop J Health Dev. 2013;27(3):192-9.

20. Paudel D: Trends and determinants of neonatal mortality in Nepal: further analysis of the Nepal demographic and health survey, 2001-2011: Ministry of Health and Population; 2013.

21. Kumar GA, Dandona R, Chaman P, Singh P, Dandona L. A population-based study of neonatal mortality and maternal care utilization in the Indian state of Bihar. BMC Pregnancy Childbirth. 2014;14(1):357.

22. Koffi AK, Mleme T, Nsona H, Banda B, Amouzou A, Kalter HD. Social autopsy of neonatal mortality suggests needed improvements in maternal and neonatal interventions in Balaka and Salima districts of Malawi. J Glob Health. 2015;5(1). https://doi.org/10.7189/jogh.05.010416.

23. Kananura RM, Tetui M, Mutebi A, Bua JN, Waiswa P, Kiwanuka SN, Ekirapa-Kiracho E, Makumbi F. The neonatal mortality and its determinants in rural communities of Eastern Uganda. Reprod Health. 2016;13(1):13.

24. Dahiru T. Determinants of early neonatal mortality in Nigeria: results from 2013 Nigeria DHS. Journal of Pediatrics \& Neonatal Care. 2015;2:00089.

25. Ayaz A, Saleem S. Neonatal mortality and prevalence of practices for newborn care in a squatter settlement of Karachi, Pakistan: a cross-sectional study. PLoS One. 2010;5(11):e13783.

26. Arunda $\mathrm{M}$, Emmelin A, Asamoah BO. Effectiveness of antenatal care services in reducing neonatal mortality in Kenya: analysis of national survey data. Glob Health Action. 2017;10(1):1328796.

27. Al-Ani ZR, Al-Hiali SJ, Al-Mashhadani WS. Perinatal mortality rate in Al-Ramadi Maternity and Children's Hospital, western Iraq. Saudi Med J. 2009;30(10):1296-300.

28. Yego F, D'Este C, Byles J, Nyongesa P. Williams JS. A case-control study of risk factors for fetal and early neonatal deaths in a tertiary hospital in Kenya. BMC Pregnancy Childbirth. 2014;14(1):389.

29. Shah R, Sharma B, Khanal V, Pandey UK, Vishwokarma A, Malla DK. Factors associated with neonatal deaths in Chitwan district of Nepal. BMC Res Notes. 2015;8(1):818.

30. Nascimento RMD, Leite AJM, NMGSd A, PCd A, CFd S. Determinants of neonatal mortality: a case-control study in Fortaleza, Ceará State, Brazil. Cad Saude Publica. 2012;28(3):559-72.

31. Kolola T, Ekubay M, Tesfa E, Morka W. Determinants of neonatal mortality in North Shoa Zone, Amhara Regional State, Ethiopia. PLoS One. 2016;11(10):e0164472.

32. Worku B, Kassie A, Mekasha A, Tilahun B, Worku A. Predictors of early neonatal mortality at a neonatal intensive care unit of a specialized referral teaching hospital in Ethiopia. Ethiop J Health Dev. 2012;26(3):200-7.

33. Worku AG, Yalew AW, Afework MF. The contributions of maternity care to reducing adverse pregnancy outcomes: a cohort study in Dabat District, Northwest Ethiopia. Matern Child Health J. 2014;18(6):1336-44.

34. Ndombo PK, Ekei QM, Tochie JN, Temgoua MN, Angong FTE, Ntock FN, Mbuagbaw L. A cohort analysis of neonatal hospital mortality rate and predictors of neonatal mortality in a sub-urban hospital of Cameroon. Ital J Pediatr. 2017:43(1):52.

35. Debelew GT, Afework MF, Yalew AW. Determinants and causes of neonatal mortality in Jimma zone, southwest Ethiopia: a multilevel analysis of prospective follow up study. PLoS One. 2014;9(9):e107184.

36. Diallo A, Meda N, Ouedraogo W, Cousens S, Tylleskar T. A prospective study on neonatal mortality and its predictors in a rural area in Burkina Faso: can MDG-4 be met by 2015? J Perinatol. 2011;31(10):656-63.

37. Doctor HV. Assessing antenatal care and newborn survival in sub-Saharan Africa within the context of renewed commitments to save newborn lives. AIMS PUBLIC HEALTH. 2016;3(3):432-47.

38. Vogel JP, Habib NA, Souza JP, Gülmezoglu AM, Dowswell T, Carroli G, Baageel HS, Lumbiganon P, Piaggio G, Oladapo OT. Antenatal care packages with reduced visits and perinatal mortality: a secondary analysis of the WHO Antenatal Care Trial. Reprod Health. 2013;10(1):19.

39. Lucas AO, Stoll BJ, Bale JR. Improving birth outcomes: meeting the challenge in the developing world. Washington, D.C.: National Academies Press; 2003.

40. Bloom SS, Lippeveld T, Wypij D. Does antenatal care make a difference to safe delivery? A study in urban Uttar Pradesh, India. Health Policy Plan. 1999;14(1):38-48.

41. Al-Ateeq MA, Al-Rusaiess AA. Health education during antenatal care: the need for more. Int J Women's Health. 2015;7:239.

42. UNICEF: UNICEF data: monitoring the situation of children and women available from https://webcache. googleusercontent.com/search?q=cache:xLTqZdN1qEIJ:https://data.unicef.org/topimaternal-health/antenatal-care/+\&cd= 1\&hl=om\&ct=clnk\&gl=et. In.; Updated: Apr 2017. 